\title{
Comparison of cognitive performance in subjects high and low in anxiety and depression
}

\author{
VLADIMIR PISHKIN, STEVEN M. FISHKIN, and WILLIAM R. LOVALLO \\ Veterans Administration Hospital and University of Oklahoma Health Sciences Center \\ Oklahoma City, Oklahoma 73104
}

\begin{abstract}
The experiment compared 72 hospitalized psychoneurotics and/or alcoholics, who were high in depression and anxiety, or low on these measures, on ability to solve a concept-identification problem. Those who were high in depression and anxiety showed fewer errors to solution of the problem than those who were low. This finding was not attributable to differences in years of education, drug regimen, or any apparent demographic variables.
\end{abstract}

The present findings were obtained as a part of a broader, long-term investigation comparing the mood and cognitive effects of two antianxiety drugs. Results dealing with the drug effects will be presented elsewhere (Pishkin, Fishkin, Shurley, Lawrence, \& Lovallo, in press). However, it is noteworthy that preliminary analysis of drug data reveals no main drug effect upon cognitive performance. The present paper concerns the relationship between cognitive performance and anxiety and depression. The results of this study were unpredicted and are apparently incompatible with a great deal of literature in this field.

\section{METHOD}

The two drug groups were 48 male, hospitalized psychoneurotics having a primary diagnosis of anxiety reaction, depression, or mixed anxiety and depression. Many of these patients had a secondary diagnosis of alcoholism. The controls were 24 hospitalized alcoholics, many of whom had a secondary diagnosis of anxiety or depression. One of the drug groups was treated for 2 weeks prior to testing with chlordiazepoxide. The other drug group was treated with doxepin for the same period of time. Control subjects received no drug. At the end of the 2-week period, all subjects were administered the Zung (1965) Self-Rating Depression Scale (SDS), an abbreviated Taylor (1953) Manifest Anxiety Scale (MAS), and a conceptidentification $(\mathrm{CI})$ problem. This last required the subject to learn to classify geometric patterns into two categories on the basis of an experimenter-determined characteristic of the stimuli. Each stimulus contained a pattern consisting of one of two sizes, two shapes, two colors, and two numbers of figures. The subject's task was to determine which of these four dimensions were relevant and then, ignoring the irrelevant ones, to classify the objects correctly and indicate his response by pressing one

This investigation was supported in part by Veterans Administration Research Service funds, MRIS 7089-07, and by a grant from the Laboratories Division, Pfizer Corporation, New York, who also supplied the drugs utilized. The authors wish to acknowledge the valuable assistance in patient evaluation and data analysis from James C. Spalding, MD, Archie B. Blackburn, MD, Robert G. Lenk, Monte L. Stahl, Andrew Karim, Gayle A. Pishkin, Jay T. Shurley, MD, and Betsy E. Lawrence, PhD. Reprint requests should be sent to Vladimir Pishkin (151 A), Veterans Administration Hospital, 921 N.E. 13th Street, Oklahoma City, Oklahoma 73104. of two buttons. For example, if size was relevant, the subject might be required to press the left button when the patterns were "large" and the right button when they were "small." A thorough description of the methodology is presented elsewhere (Pishkin, Shurley, \& Wolfgang, 1967).

\section{RESULTS AND DISCUSSION}

The data analysis compared the CI performance of the subjects who were above the median for their group on the MAS and SDS with that of those who were below the median (Pishkin et al., in press). On both tests the range of scores and the medians were essentially equivalent for all three groups of subjects. Scores on the SDS were highly correlated with those on the MAS $(r=.714, p<.005)$. This was not unexpected in that both of these tests tap, in part, a general level of neuroticism in subjects. The most surprising result was that subjects above the median in anxiety showed fewer errors to solution of the CI problem than those below the median [means $=37.3$ and 80.8 errors, respectively; $t(70)=3.27, p<.001]$. Similarly, subjects high in depression showed better CI performance than those low in depression [means $=39.8$ and 74.6, respectively; $t(70)=2.58, p<.02]$. The same general results held for the groups examined individually. In each of the three groups, when subjects were divided on the basis of a median split on either psychological test, the mean CI errors was lower for subjects above the median than for those below the median.

We attempted to explain the performance differences on the basis of differences in years of education; however, this variable failed to show any strong relationship to errors during $\mathrm{CI}(\mathrm{r}=-.1)$. Furthermore, subjects high in anxiety were slightly less educated than those low in anxiety [means $=11.3$ and 12.1 , respectively; $t(70)=1.26, p>.05]$.

Theoretically, the finding may reflect adjustive rather than maladjustive aspects of anxiety and depression and their role in information processing of psychoneurotic patients.

The finding that CI performance is better for persons 
having greater levels of anxiety may be understandable in the context of an optimal level of arousal hypothesis. This would be reasonable if our subjects who were above the median on the MAS were in fact closer to the optimal level of arousal than those subjects who fell below the median.

The finding with respect to the depression scale is more difficult to interpret. It should be mentioned first that our above-the-median subjects on the SDS scale were not exceptionally high in depression and might best be characterized as moderately depressed. This statement is based on the findings reported by Zung (1967) that a group of depressed hospitalized patients had a mean SDS index of 74 and a depressed outpatient group had an index of 64 . Our "high" subjects had SDS index scores of 56-58 and seemed to fall between Zung's depressed outpatient group and a set of groups consisting of anxiety reactions, personality disorders, and transient situational adjustment reactions. Our below-the-median subjects had mean SDS scores of $35-40$ and were not very different from a group of Zung's controls whose mean score was 33 . Since our subject groups may be characterized as exhibiting moderate and low-to-normal degrees of depressive symptomatology, the question becomes why do moderately depressed individuals perform better on a CI test than persons showing low-to-normal levels of depressed symptomatology? While we do not have an answer to this question, our speculations at this point are that: (1) individuals willing to admit negative characteristics of themselves may be healthier and more capable than those unwilling to do so, and/or (2) there may be a third factor (e.g., neuroticism) related to both anxiety and depression that is tapped by these scales and that is positively (or more probably nonlinearly) related to cognitive functioning. It is anticipated that further work currently underway in our laboratory will clarify these matters.

\section{REFERENCES}

Pishkin, V., Shurley, J. T., \& Wolfgang, A. Psychophysiological and cognitive indices in an acute double-blind study with hydroxyzine in psychiatric patients. Archives of General Psychiatry, 1967, 16, 471-478.

Pishikin, V., Fishinin, S. M., Shurley, J. T., Lawrence, B. E., \& Lovallo, W. R. Cognitive and psychophysiologic response to doxepin and chlordiazepoxide. Comprehensive Psychiatry, in press.

TAYLOR, J. A personality scale of manifest anxiety. Journal of Abnormal and Social Psychology, 1953, 48, 2.

Zung, W. W. K. A self-rating depression scale. Archives of General Psychiatry, 1965, 12, 63.

Zung, W. W. K. The measurement of depression. Manual distributed by Lakeside Laboratories, Inc., Milwaukee, Wisconsin, 1967.

(Received for publication January 10, 1978.) 